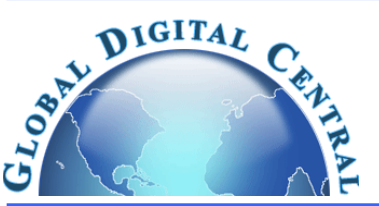

Frontiers in Heat and Mass Transfer

\title{
NUMERICAL INVESTIGATION OF FILM COOLING WITH CHEMICAL HEAT SINK
}

\author{
Keyong Chenga, b, *, Chunzi Zhang ${ }^{\mathrm{c}}$, Wei Chen ${ }^{\mathrm{a}, \mathrm{b}}$, Shiqiang Liang ${ }^{\mathrm{a}, \dagger}$, Yongxian Guo ${ }^{\mathrm{a}, \mathrm{d}}$, Xiulan Huai ${ }^{\mathrm{a}}$ \\ ${ }^{a}$ Institute of Engineering Thermophysics, Chinese Academy of Sciences, Beijing, 100190, China \\ ${ }^{b}$ Graduate University of Chinese Academy of Sciences, Beijing, 100049, China \\ ${ }^{c}$ Department of Mechanical Engineering, University of Saskatchewan, 57 Campus Drive, Saskatoon, SK S7N 5A9, Canada \\ ${ }^{d}$ School of Mechano-electronic Engineering, Xidian University, Xi'an, Shaanxi, 710071, China
}

\begin{abstract}
A film cooling method with chemical heat sink for gas turbine blades is proposed. In this method, an endothermic reaction of cooling stream occurs due to the heating from the mainstream, which leads to an improvement of film cooling effectiveness. The proposed method at different blowing ratios are computed and compared with the conventional one. The simulation result shows that due to the exsitence of the chemical heat sink the proposed method can enhance film cooling effectiveness not only in the streamwise direction, but also in the spanwise direction.
\end{abstract}

Keywords: Endothermic reaction, Heat sink, Turbine blade, Film cooling effectiveness

\section{INTRODUCTION}

Thermodynamic analysis on gas turbine show that thermal efficiency and power output can be increased with higher turbine inlet temperature (Silieti et al., 2009). At present, so high operating temperature which is far above the permissible metal temperature (Garg, 2000), has made that the cooling of blades is thus indispensable to increase the component life and reduce maintenance costs. Currently, turbine blades are cooled both internally and externally, including film cooling, jet impingement cooling, rib-turbulated cooling, pin-fin cooling, and "film + ribbed wall + impingement + pin-fin" compound cooling (Han et al., 2000).

Film cooling represents one of the few changing technologies that have allowed the achievement of today's high firing temperature, highefficiency gas turbine engines (Eriksen and Goldstein, 1974). Therefore, many investigations, focusing on injection angles of cooling stream, injection holes configuration, blowing ratios et al., have been conducted to improve the performance of film cooling. Kercher (1998) presented an exhaustive list of film cooling literatures. The most basic film cooling geometry consists of a row of round holes on a flat plate and is the most realistic simplified geometry for turbine film cooling, but this kind of cooling structure has an obviously jet lift-off behavior. Therefore, shaped film cooling holes have been typically used to combat the jet lift-off behavior. Bunker (2005) provided an excellent overview for literatures involving the shaped film cooling holes, and examined the origins of shaped film cooling and summarized the extant literature knowledge concerning the performance of such film cooling holes. Many other film cooling designs have been proposed over the years to improve film cooling effectiveness. Kusterer et al. (2007) presented a high film-cooling effectiveness as a result of the welldesigned cooling holes arrangement for interaction of two neighboring cooling jets (Double-jet Film-cooling, DJFC). The results showed that the improved film cooling effectiveness could be reached because an anti-kidney vortex pair is established in the double-jet. Heidmann and Ekkad (2008) proposed an anti-vortex turbine film cooling holes concept. Unlike shaped film cooling holes and other cooling structures, it only requires easily machinable round holes. Detailed flow visualization showed that as expected, the design could counteract the detrimental vorticity of the round holes flow, and allow it to remain attached to the surface. Sargison et al. (2002) experimentally investigated the performances of the converging slot-hole or console film-cooling holes geometry. The console film-cooling hole geometry offers advantages to the engine designer due to a superior aerodynamic efficiency over the fan-shaped holes geometry. Jia et al. (2003) conducted the numerical simulations and experiments on a slot film cooling configuration with various blowing ratios and angles. The results showed that the $30-\mathrm{deg}$ jet could provide the highest film cooling effectiveness in all the tested blowing angles, and the appropriate blowing ratio was around 1.0. It was also concluded that the existence of the recirculation only depended on the jet angle, but the size of the recirculation depended on the cross-to-jet flow ratio. Bunker (2002) had done experiments to examine two film cooling geometries which were formed by the combination of internal discrete film holes feeding continuous 2D surface slots. The holes-within-slot film effectiveness data were compared with both axial and radial film effectiveness data obtained in the same test section. The holes-in-slot geometries demonstrated two important characteristics: (1) a relative insensitivity of the adiabatic film effectiveness to blowing ratios; (2) no observed film blow-off at high blowing ratios.

As next-generation turbines will be required to burn alternate fuels, the operation temperature will increase ( $\mathrm{Li}$ and Wang, 2006). Furthermore, cooling stream can't be supplied unlimitedly (Burdet, 2009). Although the conventional cooling methods have significantly contributed to cooling enhancements in the past, the increased benefits are about to approach their limits. Therefore, the developments of advanced cooling methods with high efficiency have become one of the main supporting technologies in high-performance aircraft engines and gas turbines.

In 1997, from a second look at the mechanism of convective heat transfer, Guo et al. (1998) introduced a novel concept of heat transfer enhancement. Subsequently, an analog between heat conduction and

\footnotetext{
* Currently in Institute of Engineering Thermophysics, Chinese Academy of Sciences, Beijing, 100190, China

† Corresponding author. Email: lsq@iet.cn
} 
convection with heat sources was made to have a further understanding of the novel concept. It led to the following equation through merging the energy equations of heat conduction and convection.

$\int_{0}^{\delta_{t}} \rho C_{p}\left(u \frac{\partial T}{\partial x}+v \frac{\partial T}{\partial y}\right) d y=-k \frac{\partial T}{\partial y}$

From Eq.(1), it is easy to find that the convective term in the energy equation for the boundary layer flow corresponds to the heat source term in the heat conduction equation. The wall heat flux is equal to the overall strength of heat source inside the thermal boundary layer. This implies that the convective heat transfer can be enhanced (or weakened) by raising (or reducing) the value of the integral of convection term-heat source (or heat sink) over the thermal boundary layer. In a word, when the wall is heated by fluid, heat source enhances convective heat transfer, and heat sink weakens convective heat transfer.

Based on the theory of heat transfer enhancement, a film cooling method with chemical heat sink is proposed in the present work. First of all, cooling stream $\mathrm{C}$ is heated by the aircraft body, and ejected through discrete holes on the blade exterior surface. At the same time, cooling stream $\mathrm{C}$ absorbs the heating from the mainstream, and the endothermic reaction $(\mathrm{C} \rightarrow \mathrm{B}+\mathrm{A})$ is triggered inside the protective film boundary layer. When the chemical reaction occurs, the reaction products $\mathrm{A}$ and B spread on the surface of blades and form a protection layer that can prevent the heating of the mainstream from entering into the blades. The heating absorbed in the reaction is equivalent to the internal heat source term (heat sink) added to the energy equation of convective heat transfer. For this reason, heat transfer is weakened, and film cooling effectiveness is enhanced.

In the present study, a film cooling method with chemical heat sink is numerically computed and compared to the conventional method at different blowing ratios. This paper is aimed to investigate the potential of film cooling effectiveness enhancement compared with the conventional methods.

\section{PHYSICAL MODEL}

\subsection{Geometry}

Figure 1 shows the schematic diagram of computational domain. The computational domain includes the cooling stream supply channel, round cooling hole, and the mainstream channel. The diameter of the cylindrical part of the cooling hole, D is $12.7 \mathrm{~mm}$ with an inclination angle of $\alpha=30^{\circ}$ injecting in the mainstream channel, and the ratio of the length of the cooling hole to the diameter of the cooling hole is 4 . This paper is presented to testify the feasibility of the proposed method, and it is not necessary to adopt the complicated geometry for the simulation. Therefore, there is only one hole in the present study, and the distance from the center of the film hole to two borders is $1.5 \mathrm{D}$. The sizes of the mainstream channel are $622.3 \mathrm{~mm}$ in length, $38.1 \mathrm{~mm}$ in width and $127 \mathrm{~mm}$ in height, and the sizes of cooling tank are 100 $\mathrm{mm}$ in length, $38.1 \mathrm{~mm}$ in width and $50 \mathrm{~mm}$ in height.

\subsection{Boundary Conditions}

The normal speed of the mainstream is set to $20 \mathrm{~m} / \mathrm{s}$. For simulation of film cooling effect, the temperature of the mainstream maintains as 300 $\mathrm{K}$, and the temperature of the cooling stream is set to $150 \mathrm{~K}$. In addition, the turbulence intensity at the inlet boundary of the mainstream is $2 \%$. The same turbulence intensity is set at inlet boundary of cooling stream. The velocity of cooling stream can be determined by the blowing ratio (Nasir, et al., 2003) which is defined as below:

$M=\frac{\rho_{c} U_{c}}{\rho_{g} U_{g}}$

where $\rho$ and $U$ are the density and velocity respectively, and the subscripts $g$ and $c$ represent the mainstream and the cooling stream, respectively. The blowing ratios used in this study are $0.5,1.0$ and 1.5 with the density ratio of 2.0 .

In the simulations, adiabatic and no-slip boundary conditions are used for the walls. In addition, periodic conditions at the side walls of the main channel are specified. Pressure boundary condition is specified at the exit of the mainstream flow channel. Wall-function method is taken in the near wall region. In the present method, the standard $k-\varepsilon$ model is used. The equations is discretized by adopting the second order high resolution method. The convergence criterion of the solutions is that the relative residual of the physical quantities is less than $10^{-5}$.

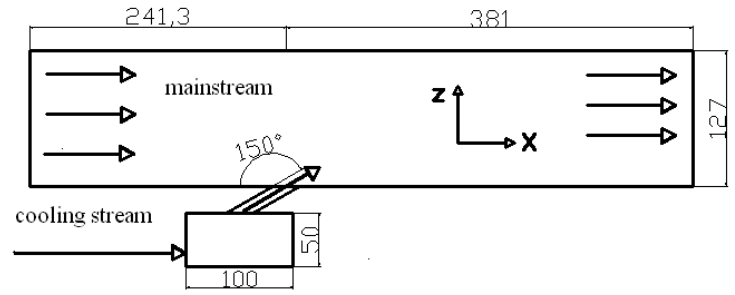

Fig. 1 Schematic diagram of computational domain

\section{GRIDS DEPENDENCY ANALYSIS}

In the present study, structured hexahedral grids are constructed for the numercial simulation. Because of the geometrical complexity of film cooling, separate grids for the different flow domains are generated by using suitable grid generator. For the mainstream domain, H-type grids are used, while the O-type grids are used to improve the orthogonality of the mesh for the round-like film cooling pipe.

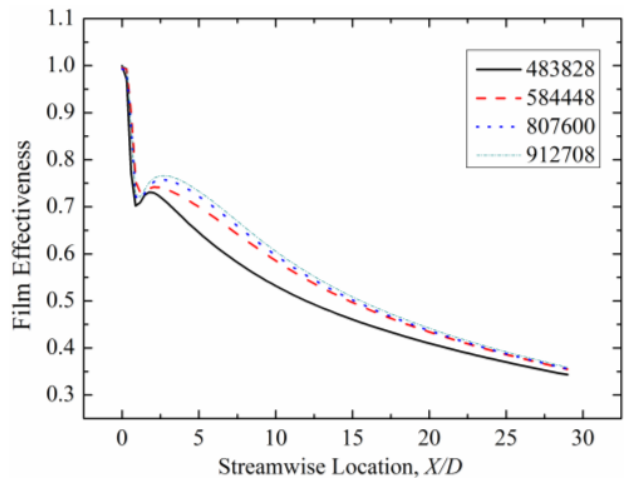

Fig. 2 The grids dependency test

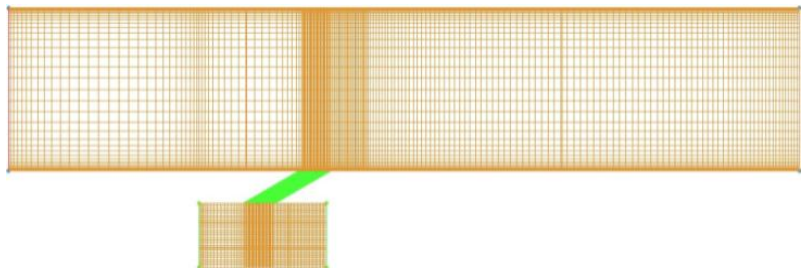

Fig. 3 Computational grids of the domain

A preliminary grid dependency test for the geometry has been carried out. Four types of node numbers, 483828, 584448, 807600 and 912708, were tested to find the optimal node numbers for the geometry shown in Fig. 2. From the figure, it could be seen that the film effectiveness of node numbers 807600 and 912708 are almost the same, 
the difference is ignored. Finally, node number, 807600, was selected. In this study, the main channel includs $200 \times 60 \times 55$ nodes $(\mathrm{x}, \mathrm{y}$, and $\mathrm{z}$ directions), and $80 \times 60 \times 27$ nodes and $30 \times 20 \times 30$ nodes are for the cooling tack and round cooling hole, respectively. Computational grids of the domain are shown in Fig. 3.

$Y^{+}$is also one of the important parameters responsible for the quality of computing grids, and the requirements for $Y^{+}$value on the first border layer are different for various turbulence models. For the standand $k$ - $\varepsilon$ model, $Y^{+}$value is need to be in $11 \sim 200$. With respect to the criteria required for the individual near wall treatment, $Y^{+}$value in the first cell adjacent to the walls was set always below 11 in the present study.

\section{RESULTS AND DISCUSSION}

The cases used in the present studies are listed in Table 1.

Table 1 Simulation cases

\begin{tabular}{|c|c|c|}
\hline Case & 1 & 2 \\
\hline Method & Conventional & Present \\
\hline Reaction formulas & None & $\mathrm{C} \rightarrow \mathrm{B}+\mathrm{A}$ \\
\hline Reaction heat & None & $100 \mathrm{~kJ} / \mathrm{kg}$ \\
\hline Reaction rate & None & $(T-200 \mathrm{~K}) / 100 \mathrm{~K}$ \\
\hline
\end{tabular}

Reaction heat is defined as the heat absorbed by kilogram of reactant during the chemical reaction. Reaction rate determines the speed of the chemical reaction per unit time. Higher reaction rate means lots of reactant take part in the chemical reaction, and aborb a large amount of heat.

The computational results are presented and compared by introducing a non-dimensional parameter-local adiabatic film cooling effectiveness (Lee and Kim, 2010), which is defined as:

$\eta=\frac{T_{g}-T_{w}}{T_{g}-T_{c}}$

Here, $T_{g}$ is the mainstream gas temperature, and remains constant. $T_{c}$ is the cooling stream temperature, and typically equals to the coolant exit bulk temperature at the injection point into the mainstream. $T_{w}$ is the adiabatic wall temperature.

\subsection{Validation}

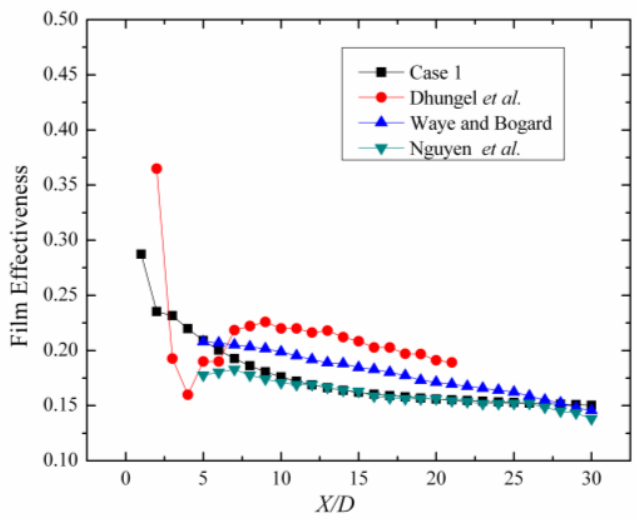

Fig. 4 Comparisons of film effectiveness with the literatures

The span-averaged film effectiveness of the cylindrical film hole for Case 1 is compared with previously published data in order to validate the current CFD model. The cylindrical geometry is selected since it is the most used baseline geometry in previous studies. As shown in Fig. 4, compared to published data by Waye and Bogard (2007), the spanaveraged film effectiveness obtained from the current simulation is underpredicted, and the maximum difference attained at the loaction of $\mathrm{X} / \mathrm{D}=14$ is $14.8 \%$, and there is no much difference compared to that by Nguyen et al. (2011). However, there are some differences between the results obtained in the current study and published data by Dhungel et al. (2009). In general, the present numerical results are closely fitted with the data in literatures, which demonstrates that the model in the present study is reliable.

\subsection{Film Cooling Effectiveness on the Bottom Surface}

Figure 5 shows the contours of film cooling effectiveness on the bottom surface of the mainstream channel. As is well known, the main factors influencing the film cooling effectiveness are spanwise spreading and streamwise fluctuation of the cooling stream. As shown in the figures, Case 2 displays not only much higher film effectiveness, but also broader jet area than Case 1 at various blowing ratios. A reason for this can be concluded that the cooling stream from the film hole forms a protective layer, and prevents the bottom wall from being heated by high temperature gas. At the same time the chemical reaction of cooling stream absorbing heat from the mainstream is triggered inside the protective film boundary layer, and film cooling effectiveness is drastically improved.

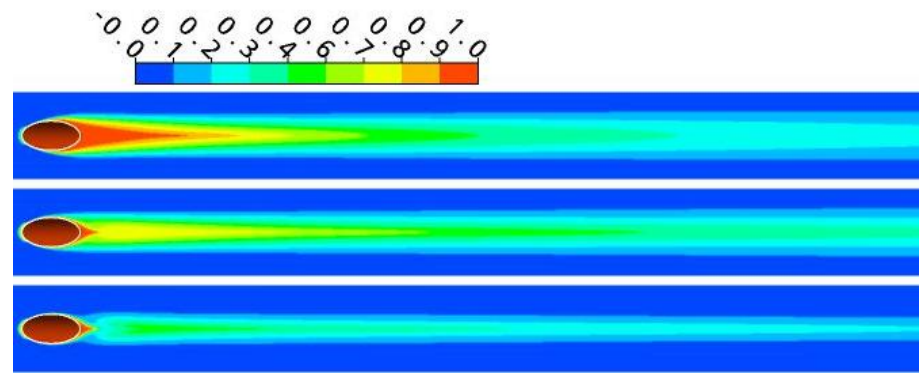

(a) Case $1(M=0.5,1.0$ and 1.5 from the top)

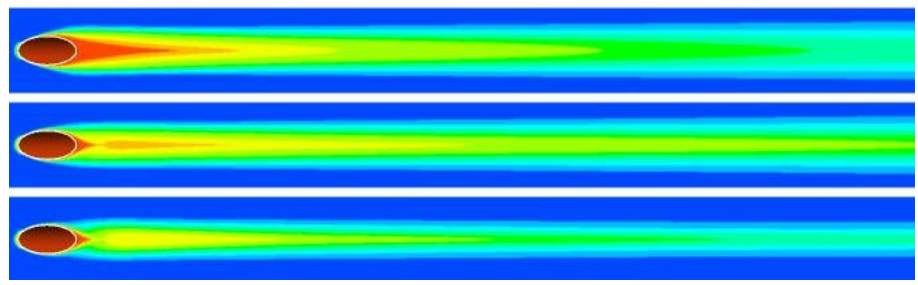

(b) Case $2(M=0.5,1.0$ and 1.5 from the top $)$

Fig. 5 Contours of film cooling effectiveness

The film fluctuation along the streamwise direction also can be found in Fig. 5. The simulation results demonstrate that both cases display the jet lift-off and reattachment at $M=1.0$ and 1.5 , but exhibit a monotonic decay for the film effectiveness at $M=0.5$. This is a consequence of the higher momentum ratio of the cooling stream. Nevertheless, it is still hard to judge whether the present film cooling with chemical heat sink influences the attachement of the jet on the bottom wall of the mainstream channel.

\subsection{Spanwise Film Cooling Effectiveness}

The most important difference between conventional film cooling 


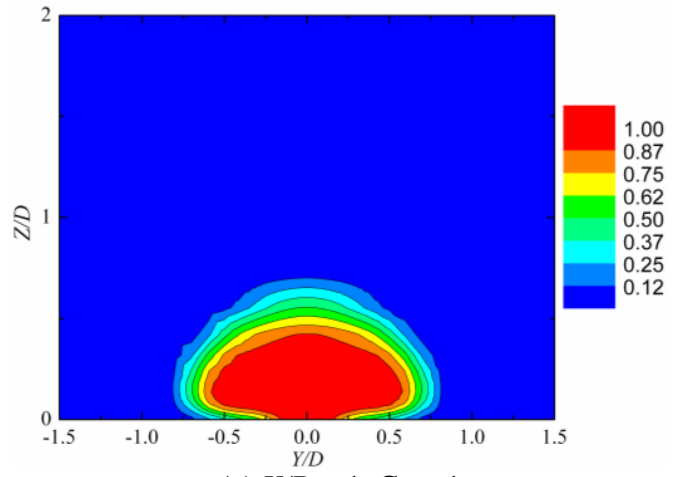

(a) $X / D=1$, Case 1

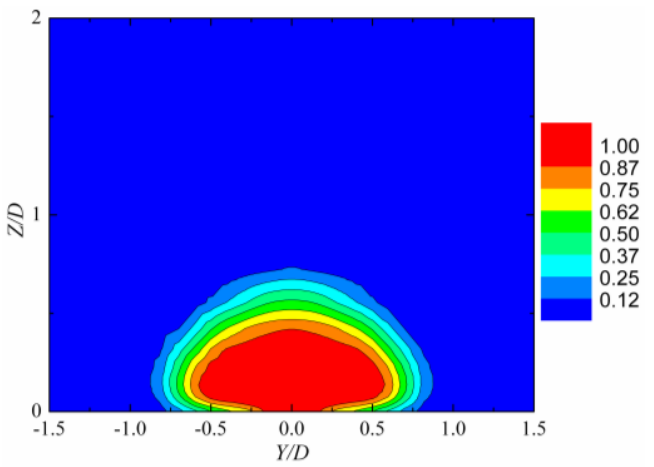

(b) $X / D=1$, Case 2

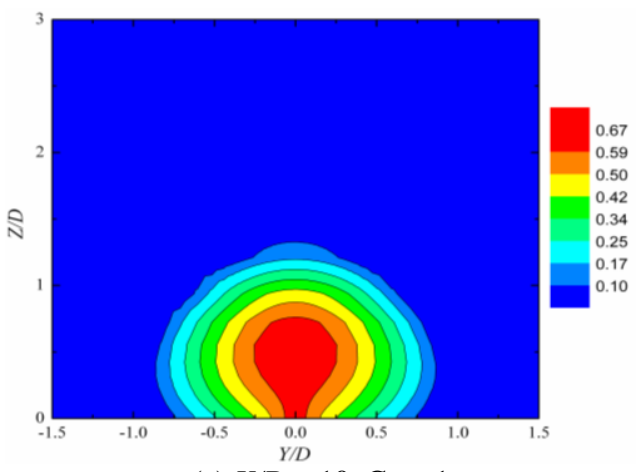

(c) $X / D=10$, Case 1

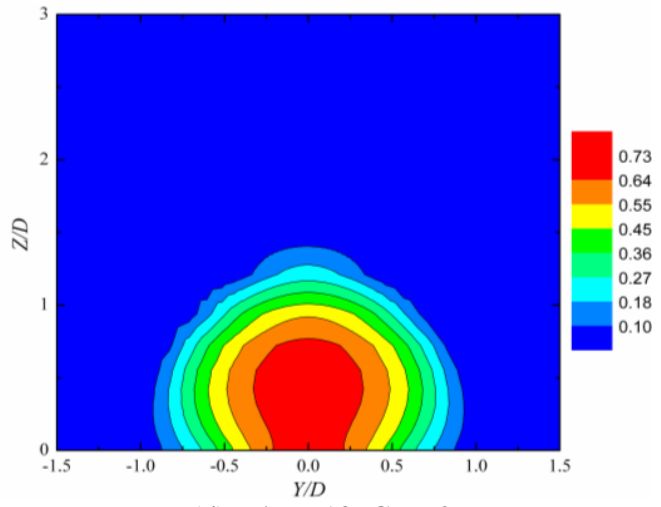

(d) $\mathrm{X} / \mathrm{D}=10$, Case 2

Fig. 6 Contour of span-wise film cooling effectiveness

methods and the present one is that the latter can produce the endothermic chemical reaction during the cooling. The present method is introduced to enhance the spanwise spreading of cooling stream and solve the consistently complained problem that for the conventional method the film cooling effectiveness decays rapidly downstream of the film hole. Figure 6 shows the contours of the film cooling effectiveness at the location of $X / D=1$ and $X / D=10$ at $M=1.0$. Note that $X=0$ is located at the center of the film hole. From the figures, it can be clearly seen that for Case 2 the spanwise coverage of the cooling stream is about $1.6 \mathrm{D}$ at $X / D=1$, and about $1.7 \mathrm{D}$ at $X / D=10$, being increased approximately $2 \mathrm{D}$ compared to Case 1 respectively. Therefore, Case 2 displays much higher film effectiveness in the spanwise direction. It also can be seen from the figures that the expanding area of the cooling stream in the present method along the Z-axis is bigger than that for Case 1, and this enhances the strength of fighting against the mainstream.

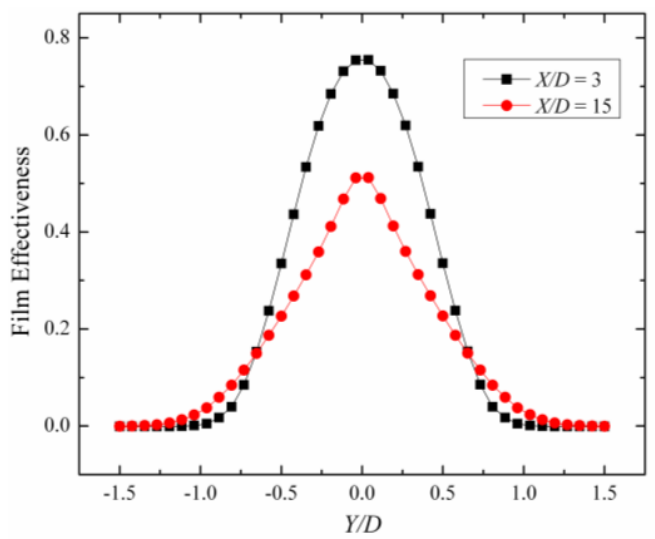

(a) Case 1

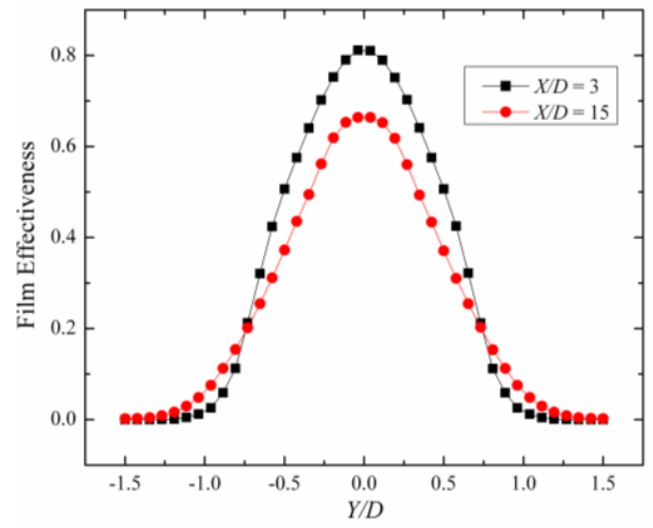

(b) Case 2

Fig. 7 Distributions of spanwise film cooling effectiveness

The distributions of spanwise film cooling effectiveness at the location of $X / D=3$ and $X / D=15$ are plotted for two cases when the blowing ratio is 1.0, as shown in Fig. 7. For the present method, when the blowing ratio is 1.0, the spanwise film effectiveness on the centerline $(Y / D=0)$ is decreased from 0.81 at $X / D=3$ to 0.66 at $X / D=$ 15. The drop amplitude of the spanwise film effectiveness is only $22.73 \%$. But for the conventional method, the film effectiveness is decreased from 0.75 to 0.51 , and the drop amplitude reaches to $47.06 \%$. Therefore, it can be concluded that the cooling effectiveness decays more slowly for the present method due to the existence of the chemical heat sink. It is also clearly visible that no matter the novel method or the conventional one, the spanwise coverage of the cooling stream is increased with the increase of $X / D$.

\subsection{Span-averaged Film Effectivenes}

Figure 8 shows the span-averaged film effectiveness downstream 
of the film hole for Case 1 and Case 2 at different blowing ratios. As shown in the figure, compared with the conventional film cooling method, the present one provides higher film effectiveness. At the location of $X / D=29$, the film effectiveness are improved by $59.80 \%$, $63.37 \%$, and $76.47 \%$ at $M=0.5,1.0$ and 1.5 , respectively. Therefore, it can be concluded that the film effectiveness for Case 2 is drastically improved at $M=1.5$, but the overall value is lower than that at $M=1.0$ and 0.5 . From the figure, it also can be seen that for Case 2 the highest film effeteciveness is attained at $M=0.5$ before the location of $X / D=$ 15 , and at $M=1.0$ after the location of $X / D=15$. The reason for this phenomenon may be that the cooling stream has the lift-off behavior at the exit of the film hole at sufficiently higher blowin ratio. In this study, the cooling stream always attaches to the bottom surface at $M=0.5$. However, with the increase of the blowing ratio the well-known counter-rotating vortex pair causes the cooling stream to separate from the bottom surafce. Therefore, Case 2 shows the best performance at $M$ $=0.5$ before the location of $X / D=15$. But the cooling stream reattaches to the bottom surface with the increase of $X / D$ at $M=1.0$, and Case 2 attains the highest film effectiveness after the location of $X / D=15$.

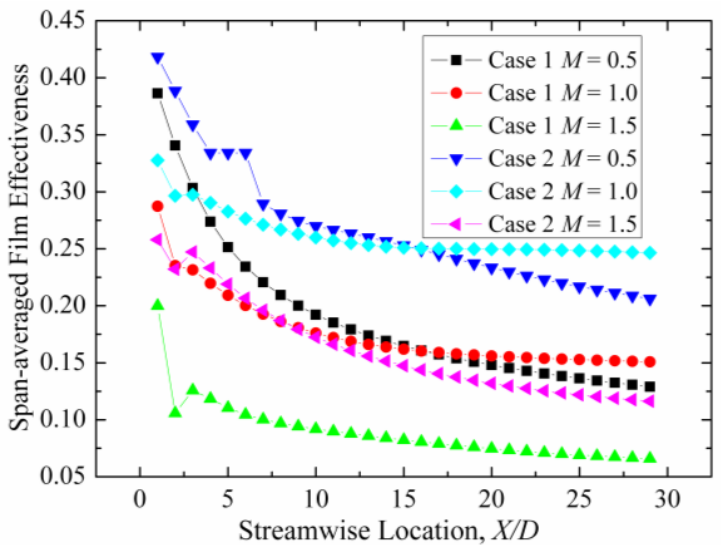

Fig. 8 Span-averaged film effectiveness

\subsection{Contours of Mass Fraction of the Cooling Stream}

Contours of mass fraction of the cooling stream on the $\mathrm{X}-\mathrm{Z}$ plane $(Y=0)$ are shown in Fig. 9. As shown in the figure, the reactions for most of the cooling stream take place not far from the film hole at low blowing ratio. With the increase of the blowing ratio, the higher velocity of the cooling stream makes the reactions delay along the X-axis. It also can be found that the cooling stream always attaches to the bottom surface at low blowing ratio, and with the increase of the blowing ratio, the cooling stream lifts off and reattaches to the bottom surface. However, at $M=1.5$ it is always sperated from the bottom surface.

\section{CONCLUSIONS}

In the present work, based on the theory of heat transfer enhancement, a film cooling method with chemical sink is proposed to improve the film cooling effectiveness of gas turbine blades. Film cooling effectiveness at different blowing ratios are numerically computed and compared with the results for the conventional methods. The simulation results indicate that compared with the conventional method, the proposed method demonstrates not only much higher film effectiveness, but also broader jet area, and the span-averaged film effectiveness are improved by $59.80 \%, 63.37 \%$, and $76.47 \%$ at $M=0.5,1.0$ and 1.5 , respectively, and the film effectiveness decays slowly along the X-axis. The present method still has the lift-off phenomenon, and the reaction of the cooling stream is affected by the blowing ratio.

At present, the most important thing is to find out a kind of suitable cooling stream medium. When this medium is heated by main stream, endothermic reaction is triggered. $\mathrm{NH}_{3}$ is considered to be used to cooling turbine blades as cooling stream. Up to now, there is still many work need to be done to study the effects of various parameters on the cooling performance of the present method.

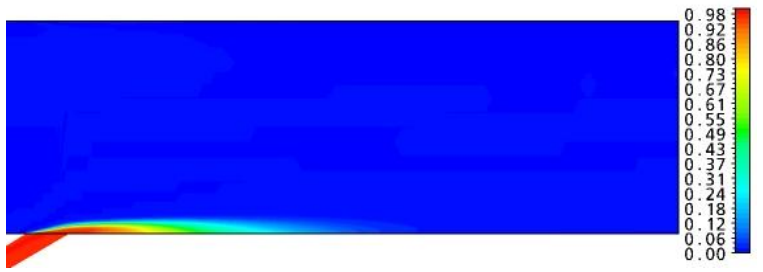

(a) $M=0.5$

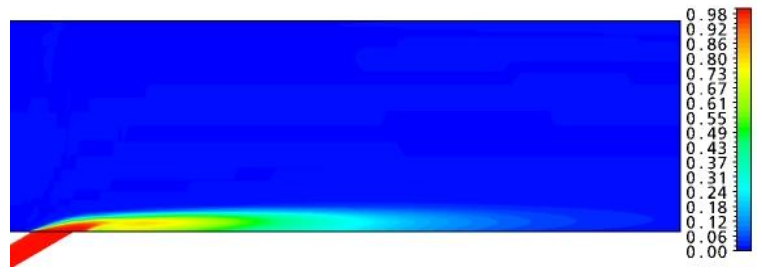

(b) $M=1.0$

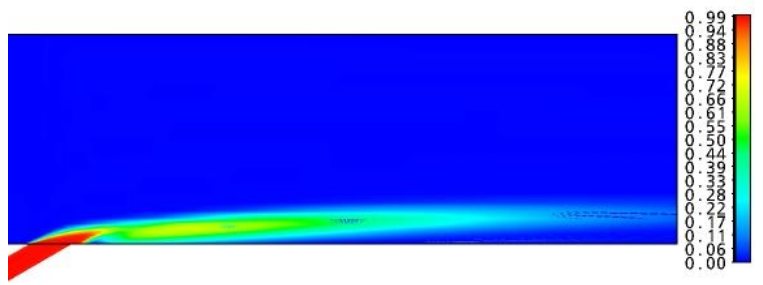

(c) $M=1.5$

Fig. 9 Mass fraction of the cooling stream

\section{ACKNOWLEDGEMENTS}

This work is supported by the National Natural Science Fundation of China under Grant No.50976118 and the National Basic Research Program of China (973 Program) under Grant No. G2010CB227302. Assistances from Associate Professor Jun Cai and Dr. Xunfeng Li for this study are greatly appreciated.

\section{NOMENCLATURE}

M Blowing ratio

$T \quad$ Temperature (K)

$D \quad$ Diameter (mm)

$U \quad$ Velocity $(\mathrm{m} / \mathrm{s})$

$Y^{+} \quad$ The value of near-wall spacing

$X \quad$ Coordinate $(\mathrm{mm})$

$Y \quad$ Coordinate $(\mathrm{mm})$

$\mathrm{Z} \quad$ Coordinate $(\mathrm{mm})$

Greek Symbols

$\rho \quad$ density $\left(\mathrm{kg} / \mathrm{m}^{3}\right)$

$\eta \quad$ Film cooling effectiveness

Subscripts

$g \quad$ Mainstream

$c \quad$ Cooling stream

w Wall 


\section{REFFERENCES}

Bunker, R. S., 2002, "Film Cooling Effectiveness Due to Discrete Holes Within a Transverse Surface Slot”, Paper No. GT2002-30178, ASME Turbo Expo 2002: Power for Land, Sea, and Air, Amsterdam, The Netherlands, June 3-6. http://dx.doi.org/10.1115/GT2002-30178

Bunker, R. S., 2005, "A review of shaped hole turbine film-cooling technology", J Heat Trans, 127(4), 441-454.

http://dx.doi.org/10.1115/ S1023621X98000062.

Burdet, A., and Abhari, R. S., 2009, "Influence of near hole pressure fluctuation on the thermal protection of a film-cooled flat plate", J Heat Trans, 131(2), 022202. http://dx.doi.org/10.1115/1.2995651.

Dhungel, A., Lu, Y., Phillips, W., Ekkad, S.V. and Heidmann, J., 2009, "Film Cooling From a Row of Hes Supplemenolted With Antivortex Holes", J Turbomach, 131(2), 021007. http://dx.doi.org/10.1115/1.2950059.

Eriksen, V., and Goldstein, R., 1974, "Heat transfer and film cooling following injection through inclined circular tubes", J Heat Trans, 96(2), 239-246.

http://dx.doi.org/10.1115/1.3450171.

Garg, V. K., 2000, "Heat transfer on a film-cooled rotating blade", Int J Heat Fluid Fl, 21(2), 134-145. http://dx.doi.org/10.1016/S0142-727X(99)00072-7.

Guo, Z. Y., Li, D. Y., and Wang, B. X., 1998, “A novel concept for convective heat transfer enhancement", Int J Heat Mass Trans, 41(14), 2221-2225.

http://dx.doi.org/10.1016/S0017-9310(97)00272-X.

Han, J.C., Dutta, S., and Ekkad S. V., 2000, Gas Turbine Heat Transfer and Cooling Technology, Taylor \& Francis Group, New York.

Heidmann, J. D., and Ekkad, S. V., 2008, “A Novel Antivortex Turbine Film-Cooling Hole Concept”, J Turbomach, 130(3), 031020. http://dx.doi.org/10.1115/1.2777194.

Jia, R., Sundén, B., and Miron, P., 2003, "Numerical and experimental study of the slot film cooling jet with various angles", Paper No. HT2003-47372, ASME Heat Transfer Summer Conference, Las Vegas, Nevada, USA, July 21-23.

\section{http://dx.doi.org/10.1115/HT2003-47372}

Kercher, D. M., 1998, “A Film-Cooling CFD Bibliography: 19711996”, Int J Rotating Mach, 4(1), 61-72. http://dx.doi.org/10.1155/S1023621X98000062

Kusterer, K., Bohn, D., Sugimoto, T. and Tanaka, R., 2007, "Double-jet ejection of cooling air for improved film cooling", $J$ Turbomach, 129(4), 809-816.

http://dx.doi.org/10.1115/1.2720508.

Lee, K. D., and Kim, K. Y., 2010, "Shape optimization of a fan-shaped hole to enhance film-cooling effectiveness", Int J Heat Mass Trans, 53(15-16), 2996-3005.

http://dx.doi.org/10.1016/j.ijheatmasstransfer.2010.03.032.

Li, X., and Wang, T., 2006, "Simulation of film cooling enhancement with mist injection", J Heat Trans, 128(6), 509-520. http://dx.doi.org/10.1115/GT2005-69100.

Nasir, H., Acharya, S., and Ekkad, S. V., 2003, "Improved film cooling from cylindrical angled holes with triangular tabs: effect of tab orientations", Int J Heat Fluid Fl, 24(5), 657-668. http://dx.doi.org/10.1016/S0142-727X(03)00082-1.

Nguyen, C. Q., Johnson, P. L., Bernier, B. C., Ho, S. H. and Kapat, J. S., " Comparison of film effectiveness and cooling uniformity of conical and cylindrical-shaped film hole with coolant-exit temperature correction", J Thermal Sci Eng Appl, 2011, 3(3), 031011.

http://dx.doi.org/10.1115/1.4003886

Sargison, J. E., Guo, S. M., Oldfield M. L. G., Lock, G. D. and Rawlinson, A. J., 2002, "A converging slot-hole film-cooling geometry Part 1: low-speed flat-plate heat transfer and loss", J Turbomach, 124(3), 453-461. http://dx.doi.org/10.1115/1.1459735.

Silieti, M., Kassab, A. J., and Divo, E., 2009, "Film cooling effectiveness: Comparison of adiabatic and conjugate heat transfer CFD models", Int J Therm Sci, 48(12), 2237-2248. http://dx.doi.org/10.1016/j.ijthermalsci.2009.04.007,

Waye, S. K, and Bogard, D. G., 2007, "High resolution film cooling effectiveness measurements of axial holes embedded in a transverse trench with various trench configurations", J Turbomach, 129(2), 294303. http://dx.doi.org/10.1115/1.2464141. 